\title{
Multiresolution Wavelet Denoising for Ultra-Wideband Time-of-Arrival Estimation with Regularized Least Squares ${ }^{\text {th }}$
}

\author{
Ted C.-K. Liu, Xiaodai Dong, Wu-Sheng Lu \\ Department of Electrical and Computer Engineering, University of Victoria, Victoria, \\ British Columbia V8W 3P6, Canada
}

\begin{abstract}
Improving accuracy in wireless localization and ranging is a challenging task which often demands an increase in the signal-to-noise ratio (SNR). Impulsive ultra-wideband (UWB) technology is a promising signaling alternative that is capable of high-resolution ranging with minimal cost on SNR. Unfortunately, typical UWB time-of-arrival (ToA) estimators are complicated and perform poorly in the low SNR environment. In this correspondence, we propose a regularized least squares (RLS) approach with wavelet denoising to improve the estimator accuracy at low SNR. Our approach estimates the ToA as a by-product of the RLS channel estimator based on a thresholding technique, which is simple and can enable fast, on-the-fly, accurate ToA estimation applicable to real-time application. In addition to the meticulous selection of a threshold based on the Neyman-Pearson criterion, we demonstrate the robustness of our algorithm first by computer simulation, then applying it to a realistic situation of range estimation via the UWB impulse radio (UWBIR). In both cases, our algorithm is shown to supersede other high-resolution algorithms in ToA estimation, energy capture and computational complexity when the sampling rate is available.
\end{abstract}

Key words: Ultra-wideband (UWB), impulse radio (IR), time-of-arrival

\footnotetext{
This work was supported by the Natural Sciences and Engineering Research Council (NSERC) of Canada. This work was presented in part at the Asilomar Conference on Signals, Systems, and Computers, Monterey, California, October 2008.

Email addresses: tcliu@ece.uvic.ca (Ted C.-K. Liu), xdong@ece.uvic.ca (Xiaodai Dong), wslu@ece.uvic.ca (Wu-Sheng Lu)
} 
(ToA) estimation, regularized least squares (RLS), wavelet denoising (WD), ranging, channel estimation, discrete wavelet transform (DWT)

\section{Introduction}

The U.S. Federal Communications Committee (FCC) spectrum mandate for ultra-wideband (UWB) communications in 2002 has brought forth many potential applications from industry. Particularly in ranging and localization, UWB impulse radio (UWB-IR) has shown to be a promising candidate that can enable centimeter accuracy with minimum cost on the signal-to-noise ratio (SNR) [1].

Locating a node in a wireless sensor network (WSN) involves obtaining the range information between a target node (TN) and a group of reference nodes (RNs) [1]. When the positions of the RNs are known, the whereabouts of the TN can be estimated by obtaining the ToA information from radio signals traveling between the nodes. Then, combining these ToA estimates to produce the position estimate of the TN within the network [1, 2]. For a real-time application which demands the instantaneous tracking of the TN position, the processing of information must be on the order of a fraction of a second, so a good ToA estimator not only has to guarantee ranging accuracy but also low in complexity to enable real-time signal processing.

The range information that is often embedded in the direct-path signal can be retrieved by several techniques, e.g., with signal strength, or timeof-arrival (ToA) estimation [2]. By operating with pulses containing a wide spectral support, UWB-IR enables fine time resolution that complements the time-based positioning technique as the viable solution for cost-effective, high-resolution ranging and localization. However, the wide spectrum can have a pronounced impact on estimation since the incurred multipath components (MPCs) are often stronger than the direct path, especially in a nonline-of-sight (NLOS) environment [3].

To circumvent that, Lee and Scholtz [4] first considered impulse-based ranging technique in a dense multipath environment with the generalized maximum likelihood (ML) estimator which detects the direct-path arrival while treating other MPCs as nuisance parameters. However, it complicates the matter with the statistical modeling of several parameters from measurement, all of which are subject to change depending on the environment, and apart from being an iterative algorithm it operates on Nyquist rate samples. 
To reduce the sampling rate requirement, 5] compared the symbol rate samples of an energy detector to a normalized threshold to estimate the ToA. However, because of a square-law device its performance degrades at low SNR and is subject to change unless a meaningful threshold can be found. To improve the performance, [6] introduced a two-stage ToA algorithm which combines the coarse estimate of an energy detector with the fine resolution of a low-rate correlator. By combining the two schemes, it unnecessarily increases the system complexity and defeats the purpose of sampling rate reduction intended for a square-law device. An inverse problem approach to ToA estimation was proposed by [7], where the authors estimate the ToA by treating it as a by-product of the large scale linear least squares (LS) solution. Although this algorithm is simple, the problem is ill-posed and suffers from output instability, attributable to both noise and a dense multipath, inherent of an impulsive wideband channel. Moreover, its performance is not well documented and the relationship to channel sampling rate is not examined. In all of the above contributions except [4], the performance was mostly evaluated via computer simulation with no results pertaining to the realistic ranging application. In contrast, Low et al. [8] demonstrated a FCCcompliant UWB ranging scheme in the line-of-sight (LOS) environment, and Falsi et al. [9] illustrated a set of ToA estimators based on the indoor measurement data from [3]. Both cases employ the peak-detection-based (PDB) algorithms, with a simple threshold and detect of the former to the suboptimal, high-resolution, iterative ML channel estimators in the latter. In [9], the PDB algorithms are shown to be good ToA estimators while being efficient at energy capture for both LOS and NLOS environments.

To improve the estimator accuracy at low SNR while retaining its simplicity for the ease of real-time positioning, we propose a regularized LS (RLS) approach with wavelet denoising (WD) to the problem of ToA estimation. Pioneered by Donoho and Johnstone [10], WD has been successfully applied to boost the low SNR performance of time-delay estimator [11] and several direction-of-arrival algorithms, e.g., [12, 13]. Our technique utilizes the discrete wavelet transform (DWT), and hyperbolic shrinkage of [14] with the threshold developed by Donoho [15] to effectively enhance the SNR prior to RLS channel estimation; thereafter, the final retrieval of accurate ToA information via a thresholding technique. Our approach is simple and enables fast, on-the-fly, high-resolution ToA estimation applicable to real-time ranging system. In addition to devising a threshold selection strategy based on the Neyman-Pearson (NP) criterion, we demonstrate the superior per- 
formance of our estimator first in computer simulation and then reaffirm with an application in the realistic indoor ranging. In both cases, our algorithm is shown to outperform the high-resolution, iterative PDB algorithms of [9] in aspects such as ToA estimation, energy capture and computational complexity when Nyquist sampling rate is available. To the best of authors' knowledge, this approach has yet to be adopted for UWB-IR ToA estimation. Note that higher range resolution can be achieved by interpolating from the Nyquist rate samples.

The rest of this correspondence is organized as follows: Section 2 presents the overall system model, including a description of both LS and RLS channel estimators. To examine the benefits of denoising, Section 3 describes the critical components of WD and how they contribute to the SNR enhancement. Then, we propose our ToA estimator in Section 4 , outline the threshold selection criterion in Section 5 , and compare its performance against the PDB algorithms with both computer simulation and realistic UWB-IR range estimations in Section 6. Finally, concluding remarks are given in Section 7.

\section{System Model}

The position of a sensor node is directly related to the ToA of the first multipath component. To estimate the ToA, a UWB ranging system periodically transmits sub-nanosecond pulses between the RNs and a TN of unknown distance. For a single pulse transmitted through free-space, the received signal at the TN under multipath can be modeled as

$$
r(t)=\sum_{l=0}^{L-1} \alpha_{l} w\left(t-\tau_{l}\right)+n(t),
$$

where $w(t)$ is the received pulse template of duration $T_{p}, \alpha_{l}$ and $\tau_{l}$ are the amplitude and time delay of the $l$-th multipath, $L$ is an unknown a priori which presents the number of propagation paths, $n(t)$ is the additive white Gaussian noise (AWGN) with variance $\sigma^{2}$ to account for thermal noise. The purpose of ToA estimation is to accurately acquire the direct-path, i.e., $\tau_{0}$, over an observation interval $[0, T)$.

Assuming the observation interval can be divided into $K$ equally spaced delays for $k=0,1, \ldots, K-1$, each associating with a sparse channel tap $a_{k}$. We can then simplify (1) by associating the sparse set $\left\{a_{k}\right\}_{k=0}^{K-1}$ of channel 
coefficients with uniformly delayed received pulse template $w(t-k \Delta)$ for $k=0,1, \ldots, K-1$, as

$$
r(t)=\sum_{k=0}^{K-1} a_{k} w(t-k \Delta)+n(t) .
$$

Suppose the received signal is sampled at sampling time $T_{s}$. Given time instant samples $t_{i}=(i-1) T_{s}$ for $i=1,2, \ldots, M$, (2) can be written as

$$
r\left(t_{i}\right)=\sum_{k=0}^{K-1} a_{k} w\left(t_{i}-k \Delta\right)+n\left(t_{i}\right), \quad i=1,2, \ldots, M,
$$

which in matrix notation is given by

$$
\mathbf{r}=\mathbf{W a}+\mathbf{n}=\mathbf{s}+\mathbf{n}
$$

where $\mathbf{a}=\left[a_{0}, a_{1}, \ldots, a_{K-1}\right]^{T}$ and $\mathbf{n}=\left[n\left(t_{1}\right), n\left(t_{2}\right), \ldots, n\left(t_{M}\right)\right]^{T}$ are vectors of sparse channel coefficients and the noise samples, respectively, $\mathbf{s}=\mathbf{W a}$ is the signal portion of $\mathbf{r}$, and

$$
\mathbf{W}=\left[\begin{array}{cccc}
w\left(t_{1}\right) & w\left(t_{1}-\Delta\right) & \ldots & w\left(t_{1}-(K-1) \Delta\right) \\
w\left(t_{2}\right) & w\left(t_{2}-\Delta\right) & \ldots & w\left(t_{2}-(K-1) \Delta\right) \\
\vdots & \vdots & \ddots & \vdots \\
w\left(t_{M}\right) & w\left(t_{M}-\Delta\right) & \ldots & w\left(t_{M}-(K-1) \Delta\right)
\end{array}\right]
$$

represents a $M \times K$ matrix which comprises of delayed and sampled version of $w(t)$. In contrast to the matrix representations of [7] and [9], the step-size in (5) can be varied for the sake of estimation accuracy.

\subsection{LS Solution}

We treat the ToA estimation as a by-product of the LS channel estimator by solving the solution to (44). For an overdetermined noiseless system there exists a unique solution which solves the problem

$$
\min \|\mathbf{W a}-\mathbf{r}\|^{2}
$$

which yields the LS solution given by

$$
\hat{\mathbf{a}}_{\mathrm{LS}}=\left(\mathbf{W}^{T} \mathbf{W}\right)^{-1} \mathbf{W}^{T} \mathbf{r}=\mathbf{W}^{\perp} \mathbf{r},
$$


where $\|\cdot\|^{2}$ is the Euclidean norm, $(\cdot)^{T}$ denotes matrix transpose, $(\cdot)^{-1}$ is the matrix inverse, $\hat{\mathbf{a}}$ denotes an estimate of $\mathbf{a}$ and $\mathbf{W}^{\perp}$ is the MoorePenrose inverse of $\mathbf{W}$. Unlike [9], $\mathbf{W}^{\perp}$ can be pre-computed and stored when a desired resolution $\Delta$ is given. Unfortunately, (7) is often sensitive to noise in the received signal and can be quite unstable when $\mathbf{W}$ is ill-posed. In contrast, when $\mathbf{W}$ is well-posed, i.e., independent columns in $\mathbf{W}$, (7) gives the maximum likelihood estimate (MLE) of $\mathbf{a}$.

\subsection{Regularized LS Solution}

To find a meaningful result when the solution to (6) becomes unstable, we apply the technique of regularization. Regularization is a well-known technique for dealing with instability in the inverse problem [16] by forcing an ill-posed problem into a well-posed one with some a priori information. The RLS solution solves the problem

$$
\min \left\{\|\mathbf{W a}-\mathbf{r}\|^{2}+\lambda\|\mathbf{a}\|^{2}\right\},
$$

where $\lambda \geq 0$ is the regularization parameter which controls the solution's energy. Note that with $\lambda=0$ the solution to (8) reduces to the LS one. With $\lambda>0$, it is straightforward to show that the unique global solution to (8) is given by

$$
\hat{\mathbf{a}}_{\mathrm{RLS}}=\left(\mathbf{W}^{T} \mathbf{W}+\lambda \mathbf{I}\right)^{-1} \mathbf{W}^{T} \mathbf{r}=\mathbf{W}_{\lambda}^{\perp} \mathbf{r},
$$

where $\mathbf{W}_{\lambda}^{\perp}$ is called the regularized pseudo-inverse, which can also be precalculated and stored for fast, on-the-fly processing.

\section{Wavelet Denoising}

To realize a stable LS solution for accurate ToA estimation, we apply the well-established technique of WD. Since its introduction in [10], denoising with DWT has become a powerful tool to recover noise corrupted data. To recover $M$ samples of a known data sequence $\mathbf{s}$ from the noise-corrupted observation $\mathbf{r}=\mathbf{s}+\mathbf{n}$, where $\mathbf{n}$ denotes a $M \times 1$ vector samples of AWGN with variance $\sigma^{2}$, the purpose of WD is to differentiate the wavelet coefficients of $\mathbf{s}$ from those of $\mathbf{n}$, assuming the coefficients of $\mathbf{s}$ resides mostly in the low frequency region and can be compressed into a few large values in the wavelet domain. The compression is carried out by multiplying $\mathbf{r}$ with a $M \times M$ orthonormal wavelet matrix $\mathbf{W}_{W}$, as

$$
\begin{aligned}
\mathbf{r}_{W} & =\mathbf{W}_{W} \mathbf{r}=\mathbf{W}_{W} \mathbf{s}+\mathbf{W}_{W} \mathbf{n} \\
& =\mathbf{s}_{W}+\mathbf{n}_{W},
\end{aligned}
$$


where the matrix $\mathbf{W}_{W}$ can be pre-determined by knowing the wavelet filter order $F$ and decomposition level $J$. Multiplying $\mathbf{r}$ with $\mathbf{W}_{W}$ is essentially the DWT operation. Moreover, due to the orthonormal property of $\mathbf{W}_{W}$, the noise is similarly mapped to $\mathbf{n}_{W}$ with identical statistics. However, because of its wideband nature, coefficients of noise are usually small and can be discarded; whereas, the large coefficients of the desired signal are retained [10, 15]. Differentiating amongst these coefficients is identical to the filtering operation, where (10) is multiplied by a matrix $\mathbf{H}$ modeled as

$$
\mathbf{H}=\operatorname{diag}[h(1), h(2), \ldots, h(M)] .
$$

The elements of $\mathbf{H}$ are set according to the thresholding criterion, with hard and soft thresholds from [15] being the most common, or the hyperbolic shrinkage proposed by Vidakovic [14] defined as

$$
h(i)= \begin{cases}\sqrt{\left(1-\frac{\delta^{2}}{\left|r_{W}(i)\right|^{2}}\right)}, & \text { if }\left|r_{W}(i)\right|>\delta \\ 0, & \text { otherwise }\end{cases}
$$

where $r_{W}(i)$ denotes the $i$-th element of $\mathbf{r}_{W},|\cdot|$ is the absolute value operation, and $\delta$ is the threshold from [10, 15], given by

$$
\delta=\sqrt{2 \sigma^{2} \log (M)} .
$$

The recovery of the desired signal $\mathbf{s}$ is now given by

$$
\hat{\mathbf{s}}=\mathbf{W}_{W}^{T} \tilde{\mathbf{r}}_{W}=\mathbf{W}_{W}^{T} \mathbf{H} \mathbf{W}_{W} \mathbf{r},
$$

which is the inverse DWT operation to the denoised wavelet coefficients $\tilde{\mathbf{r}}_{W}=$ $\mathbf{H r}_{W}$, where $\hat{\mathbf{s}}$ represents an estimate of $\mathbf{s}$, but with the noise significantly reduced. The process of discarding and retaining the wavelet coefficients results in the overall SNR enhancement.

\section{RLS-WD ToA Estimation}

The distinct advantage of UWB in ranging is its high precision with minimal penalty on SNR. Many existing ToA estimators, however, do not work well at the low SNR region, thus are limited to only short distance ranging. To improve the estimator accuracy under low SNR, we adopt WD 
with RLS channel estimation as shown in Fig. 1 for a simple, yet accurate, ToA estimation. We name this the RLS-WD ToA estimator.

One drawback of denoising with DWT is the requirement of noise information, where its ability to remove noise depends entirely on how accurate the noise variance can be estimated. For narrowband signals and images, which map to a few large, low-frequency coefficients in the wavelet domain, noise variance can be estimated from the finest scale wavelet coefficients [15]. However, due to the wideband characteristic of UWB, estimating variance from the first level decomposition is often incorrect. For that, assuming a large distance between nodes and a large sample size $M$, the variance can be estimated from the first few hundred noise samples as

$$
\hat{\sigma}^{2}=\frac{1}{N-1} \sum_{i=1}^{N}\left(r\left(t_{i}\right)-\hat{\mu}\right)^{2},
$$

where $\hat{\mu}$ is the sample mean and $N$ a subset of $M$. Now, the RLS-WD ToA estimator can be summarized as

1. Receive $M$ samples of observation $\mathbf{r}$ at sampling rate $T_{s}$ over the interval $[0, T)$.

2. Estimate the noise variance $\hat{\sigma}^{2}$ according to (15).

3. Select the wavelet filter order $F$ and WD decomposition level $J$, apply Daubechies DWT and Vidakovic hyperbolic shrinkage to $\mathbf{r}$, and estimate the desired signal according to (14).

4. Choose the channel estimator resolution $\Delta$, and $K=T / \Delta$, construct W according to (5).

5. Estimate the channel â using either LS solution in (7), or RLS algorithm in (9), with a pre-determined $\lambda$.

6. Estimate the ToA as

$$
\hat{\tau}_{0}=\arg \min _{t_{k}}|\hat{\mathbf{a}}|>\xi,
$$

where $\xi=\eta \hat{a}_{\max }$ is the threshold, $\eta \in(0,1)$ is the normalized threshold relating to a percentage of $\hat{a}_{\max }=\max \{|\hat{\mathbf{a}}|\}$, the maximum estimated amplitude.

\section{Threshold Selection for RLS-WD Estimator}

The threshold selection plays a crucial role in the overall estimator performance. Especially in a dense multipath channel, a threshold too low can 
increase the chance that the noise samples prior to the true ToA being falsely identified as the ToA [4], also known as the early false alarm probability $P_{F A}$. Conversely, it is easy to miss the true ToA and detect the later arriving MPCs as the ToA when a threshold is set too high, thus increasing the probability of a missed detection $P_{M}$. In this section, we illustrate the threshold selection according to the NP criterion [17] and its variant, one which aims at minimizing both $P_{F A}$ and $P_{M}$ [4]. For simplicity, we do not consider the effect of $\mathrm{WD}$, and we assume the columns of $\mathbf{W}$ in (5) to be orthogonal basis functions, i.e., $\mathbf{w}_{i^{\prime}}^{T} \mathbf{w}_{j^{\prime}}=0, i^{\prime} \neq j^{\prime}$, and $\mathbf{w}_{i^{\prime}}^{T} \mathbf{w}_{i^{\prime}}=1, \forall i^{\prime}=0,1, \ldots, K-1$, where $\mathbf{w}_{i^{\prime}}$ denotes the $i^{\prime}$-th column of $\mathbf{W}$, implying non-overlapping multipaths and unit energy pulse $w(t)$. Then the $i^{\prime}$-th sample output $\hat{a}_{i^{\prime}}$ of the LS channel estimator 2 can be written as

$$
\begin{aligned}
\hat{a}_{i^{\prime}} & =\mathbf{w}_{i^{\prime}}^{T} \mathbf{w}_{i^{\prime}} a_{i^{\prime}}+\mathbf{w}_{i^{\prime}}^{T} \mathbf{n}=a_{i^{\prime}}+\sum_{i=1}^{M} w\left(t_{i}-i^{\prime} \Delta^{\prime}\right) n\left(t_{i}\right) \\
& =a_{i^{\prime}}+n_{i^{\prime}},
\end{aligned}
$$

where $\hat{a}_{i^{\prime}}$ is the MLE of $a_{i^{\prime}}, \Delta^{\prime}=\left\lfloor T_{p} / T_{s}\right\rfloor$ is the number of delay samples in between pulses to achieve orthogonality, and $\lfloor\cdot\rfloor$ denotes the floor function. Clearly, $\hat{a}_{i^{\prime}} \sim \mathcal{N}\left(a_{i^{\prime}}, \sigma^{2}\right), \forall i^{\prime}=0,1, \ldots, K-1$, is a set of non-identical but independently distributed (non-i.i.d.) Gaussian random variables 3 (r.v.'s). When the sample $\hat{a}_{i^{\prime}}$ undergoes the absolute value operation in (16), the probability density function (p.d.f.) can be written as the function of a single r.v. [18] as

$$
f_{Y_{i^{\prime}}}\left(y_{i^{\prime}}\right)= \begin{cases}0, & \text { if } y_{i^{\prime}}<0 \\ \frac{1}{\sqrt{2 \pi \sigma^{2}}}\left[\exp \left(-\frac{\left(y_{i^{\prime}}-a_{i^{\prime}}\right)^{2}}{2 \sigma^{2}}\right)+\exp \left(-\frac{\left(-y_{i^{\prime}}-a_{i^{\prime}}\right)^{2}}{2 \sigma^{2}}\right)\right], & \text { if } y_{i^{\prime}} \geq 0\end{cases}
$$

where $y_{i^{\prime}}=\left|\hat{a}_{i^{\prime}}\right|$ is the $i^{\prime}$-th output sample and $f_{Y_{i^{\prime}}}\left(y_{i^{\prime}}\right)$ is the p.d.f. of $y_{i^{\prime}}$. Therefore, following (18), the threshold of (16) with $y_{\max }:=\hat{a}_{\max }=$

\footnotetext{
${ }^{1}$ Denoising with DWT can be formulated as a special case of the treatment herein.

${ }^{2}$ The RLS channel estimator output can be treated as a special case of LS channel estimator, where $\lambda \neq 0$ in (9). The use of RLS is only necessary when the columns of $\mathbf{W}$ are not orthogonal.

${ }^{3}$ In the case when columns of $\mathbf{W}$ are not necessary orthogonal, i.e., $\mathbf{w}_{i^{\prime}}^{T} \mathbf{w}_{j^{\prime}} \neq 0, i^{\prime} \neq j^{\prime}$, we can stack the $K$ elements of $\hat{a}_{i^{\prime}}$ into vector $\hat{\mathbf{a}}=\left[\hat{a}_{0}, \hat{a}_{1}, \ldots, \hat{a}_{K-1}\right]^{T}$. The elements of $\hat{\mathbf{a}}$ is then correlated and non-identically distributed Gaussian random variable with mean $\mathbb{E}\{\hat{\mathbf{a}}\}=\left(\mathbf{W}^{T} \mathbf{W}\right)^{-1} \mathbf{W}^{T} \mathbf{W a}$ and covariance $\operatorname{Cov}(\hat{\mathbf{a}})=\sigma^{2}\left(\mathbf{W}^{T} \mathbf{W}\right)^{-1} \mathbf{W}^{T} \mathbf{W}\left(\left(\mathbf{W}^{T} \mathbf{W}\right)^{-1}\right)^{T}$.
} 
$\max \left\{y_{i^{\prime}}\right\}$, for $i^{\prime}=0,1, \ldots, K-1$, can be formulated as the order statistics [18] with its p.d.f. given as

$$
\begin{aligned}
f_{Y_{\max }}\left(y_{\max }\right)= & \sum_{i^{\prime \prime}=0}^{K-1} f_{Y_{i^{\prime \prime}}}\left(y_{\max }\right) \prod_{j^{\prime \prime} \neq i^{\prime \prime}, j^{\prime \prime}=0}^{K-1} F_{Y_{i^{\prime \prime}}}\left(y_{\max }\right) \\
= & \sum_{i^{\prime \prime}=0}^{K-1} \frac{1}{\sqrt{2 \pi \sigma^{2}}} \times \\
& \quad\left[\exp \left(-\frac{\left(y_{\max }-a_{i^{\prime \prime}}\right)^{2}}{2 \sigma^{2}}\right)+\exp \left(-\frac{\left(-y_{\max }-a_{i^{\prime \prime}}\right)^{2}}{2 \sigma^{2}}\right)\right] \times \\
& \prod_{j^{\prime \prime} \neq i^{\prime \prime}, j^{\prime \prime}=0}^{K-1}\left(Q\left(\frac{a_{i^{\prime \prime}}-y_{\max }}{\sigma}\right)-Q\left(\frac{a_{i^{\prime \prime}}+y_{\max }}{\sigma}\right)\right)
\end{aligned}
$$

where $f_{Y_{i^{\prime \prime}}}\left(y_{\max }\right)$ and $F_{Y_{i^{\prime \prime}}}\left(y_{\max }\right)$ are the p.d.f. and cumulative distribution function (c.d.f.) of $y_{i^{\prime \prime}}$ evaluated at $y_{\max }$, respectively, and $Q(x) \triangleq$ $\int_{x}^{+\infty} \frac{1}{\sqrt{2 \pi}} e^{-t^{2} / 2} \mathrm{~d} t$ is the Q-function. Now, assuming the real ToA to arrive at the $j$-th index 4 for $0 \leq j \leq K-1$ and only the signal plus noise components exist thereafter, the $P_{F A}$ can be written as

$$
\begin{aligned}
P_{F A}= & \operatorname{Pr}\left\{y_{i^{\prime}}>\xi, \text { for any } i^{\prime}=0,1, \ldots, j-1\right\} \\
= & 1-\operatorname{Pr}\left\{y_{i^{\prime}}<\eta y_{\max }, \forall i^{\prime}=0,1, \ldots, j-1\right\} \\
= & 1-\prod_{i^{\prime}=0}^{j-1}\left(1-\operatorname{Pr}\left\{y_{i^{\prime}}>\eta y_{\max }\right\}\right) \\
= & 1-\prod_{i^{\prime}=0}^{j-1}\left(1-\int_{0}^{+\infty} \int_{\eta y_{\max }}^{+\infty} f_{Y_{i^{\prime}}, Y_{\max }}\left(y_{i^{\prime}}, y_{\max }\right) \mathrm{d} y_{i^{\prime}} \mathrm{d} y_{\max }\right) \\
= & 1-\prod_{i^{\prime}=0}^{j-1} \times \\
& \quad\left(1-\int_{0}^{+\infty} \int_{\eta y_{\max }}^{+\infty} f_{Y_{i^{\prime}} \mid Y_{\max }}\left(y_{i^{\prime}} \mid y_{\max }\right) f_{Y_{\max }}\left(y_{\max }\right) \mathrm{d} y_{i^{\prime}} \mathrm{d} y_{\max }\right),(20)
\end{aligned}
$$

\footnotetext{
${ }^{4}$ We treat the real ToA that arrives in between indexes as a special case of arriving at the index.
} 
where $f_{Y_{i^{\prime}}, Y_{\max }}\left(y_{i^{\prime}}, y_{\max }\right)$ and $f_{Y_{i^{\prime}} \mid Y_{\max }}\left(y_{i^{\prime}} \mid y_{\max }\right)$ are the joint and conditional p.d.f.'s, respectively, of the r.v.'s $y_{i^{\prime}}$ and $y_{\max }$, and we have applied the Bayes' Theorem in the last step of (20). Substituting (18) into (20) gives

$$
P_{F A}=1-\prod_{i^{\prime}=0}^{j-1}\left(1-\int_{0}^{+\infty} g\left(a_{i^{\prime}}, \eta y_{\max }\right) f_{Y_{\max }}\left(y_{\max }\right) \mathrm{d} y_{\max }\right)
$$

where

$$
g(a, b)=\left(1-Q\left(\frac{a-b}{\sigma}\right)+Q\left(\frac{a+b}{\sigma}\right)\right) .
$$

For the ToA which arrives at the $j$-th index, we may assume there is no signal present for $0 \leq i^{\prime}<j$, thus $a_{i^{\prime}} \approx 0$, then (21) simplifies to

$$
P_{F A} \cong 1-\left(1-2 \int_{0}^{+\infty} Q\left(\frac{\eta y_{\max }}{\sigma}\right) f_{Y_{\max }}\left(y_{\max }\right) \mathrm{d} y_{\max }\right)^{j} .
$$

According to the NP criterion, we can set the left-hand side of (23) to a constant then look for $\eta$ by inverting the right-hand side. Unfortunately, this is not pursued here due to the complexity of inverting (23) with the evaluation of (19). Instead, we consider a simplified scenario where $\xi$ is set as a fixed threshold, then following the above formulation the $P_{F A}$ can be derived as

$$
\begin{aligned}
P_{F A} & =1-\prod_{i^{\prime}=0}^{j-1}\left(1-\operatorname{Pr}\left\{y_{i^{\prime}}>\xi\right\}\right)=1-\prod_{i^{\prime}=0}^{j-1}\left(1-g\left(a_{i^{\prime}}, \xi\right)\right) \\
& =1-\prod_{i^{\prime}=0}^{j-1}\left(Q\left(\frac{a_{i^{\prime}}-\xi}{\sigma}\right)-Q\left(\frac{a_{i^{\prime}}+\xi}{\sigma}\right)\right) \\
& \cong 1-\left(1-2 Q\left(\frac{\xi}{\sigma}\right)\right)^{j} .
\end{aligned}
$$

Following the NP criterion, we can obtain $\xi$ by inverting the right-hand side of (24) according to a target $P_{F A}$ as

$$
\xi=Q^{-1}\left(\frac{1-\left(1-P_{F A}\right)^{1 / j}}{2}\right) \sigma .
$$

Alternatively, we may set a specific $P_{F A}$ and find a threshold which minimizes the $P_{M}$, or look for a threshold which simultaneously minimizes both 
$P_{F A}$ and $P_{M}$ as in [4]. For these cases of thresholding criterion, we define $P_{M}$ as the probability that a threshold crossing is missed for the first $j+1$ samples and a false detection on any of the later $K-j-2$ signal plus noise samples, the $P_{M}$ can be formulated as

$$
\begin{aligned}
P_{M}= & \operatorname{Pr}\left\{y_{i^{\prime}}<\eta y_{\max }, \forall i^{\prime}=0,1, \ldots, j \cap\right. \\
& \left.y_{i^{\prime}}>\eta y_{\text {max }}, \text { for any } i^{\prime}=j+1, j+2, \ldots, K-1\right\} \\
= & \prod_{i^{\prime}=0}^{j} \operatorname{Pr}\left\{y_{i^{\prime}}<\eta y_{\max }\right\} \times \\
& \left(1-\operatorname{Pr}\left\{y_{i^{\prime}}<\eta y_{\text {max }}, \forall i^{\prime}=j+1, j+2, \ldots, K-1\right\}\right) .
\end{aligned}
$$

And continuing in a manner similar to the derivations of (20), we get

$$
\begin{aligned}
P_{M}= & \prod_{i^{\prime}=0}^{j}\left(1-\int_{0}^{+\infty} g\left(a_{i^{\prime}}, \eta y_{\max }\right) f_{Y_{\max }}\left(y_{\max }\right) \mathrm{d} y_{\max }\right) \times \\
& {\left[1-\prod_{i^{\prime}=j+1}^{K-1}\left(1-\int_{0}^{+\infty} g\left(a_{i^{\prime}}, \eta y_{\max }\right) f_{Y_{\max }}\left(y_{\max }\right) \mathrm{d} y_{\max }\right)\right] } \\
\cong & \left(1-2 \int_{0}^{+\infty} Q\left(\frac{\eta y_{\max }}{\sigma}\right) f_{Y_{\max }}\left(y_{\max }\right) \mathrm{d} y_{\max }\right)^{j} \times \\
& \left(1-\int_{0}^{+\infty} g\left(a_{j}, \eta y_{\max }\right) f_{Y_{\max }}\left(y_{\max }\right) \mathrm{d} y_{\max }\right) \times \\
& {\left[1-\prod_{i^{\prime}=j+1}^{K-1}\left(1-\int_{0}^{+\infty} g\left(a_{i^{\prime}}, \eta y_{\max }\right) f_{Y_{\max }}\left(y_{\max }\right) \mathrm{d} y_{\max }\right)\right] }
\end{aligned}
$$

where the last step stems from the assumption that there exists no signal for $0 \leq i^{\prime}<j$. Likewise to the evaluation of (21) or (23), looking for $\eta$ which minimizes (27) has no closed form solution due to the p.d.f. of $y_{\max }$, and one must resort to numerical integration, which can be trivially computed due to the products of the single integrals, and recognizing that the integrands would only consist of the Gaussian p.d.f.'s and Q-functions after substituting (19) and (22) into (27). Without directly computing the numerical integration, 
we assume a fixed threshold $\xi$ so that the $P_{M}$ can now be simplified as

$$
\begin{aligned}
P_{M}= & \prod_{i^{\prime}=0}^{j} \operatorname{Pr}\left\{y_{i^{\prime}}<\xi\right\} \times\left(1-\prod_{i^{\prime}=j+1}^{K-1}\left(1-\operatorname{Pr}\left\{y_{i^{\prime}}>\xi\right\}\right)\right) \\
= & \prod_{i^{\prime}=0}^{j}\left(1-g\left(a_{i^{\prime}}, \xi\right)\right) \times\left(1-\prod_{i^{\prime}=j+1}^{K-1}\left(1-g\left(a_{i^{\prime}}, \xi\right)\right)\right) \\
\cong & \left(1-2 Q\left(\frac{\xi}{\sigma}\right)\right)^{j} \times\left(Q\left(\frac{a_{j}-\xi}{\sigma}\right)-Q\left(\frac{a_{j}+\xi}{\sigma}\right)\right) \times \\
& \left(1-\prod_{i^{\prime}=j+1}^{K-1}\left(Q\left(\frac{a_{i^{\prime}}-\xi}{\sigma}\right)-Q\left(\frac{a_{i^{\prime}}+\xi}{\sigma}\right)\right)\right),
\end{aligned}
$$

which can be evaluated with $\xi$ from (25) when a particular $P_{F A}$ is chosen, or minimizing $P_{F A}$ and $P_{M}$ altogether for a specific $\xi$. We compare the theoretical derivations of $P_{F A}$ and $P_{M}$ with simulation in Fig. 2 as a function of SNR with two fixed threshold $\xi$ of $0.05 y_{\max }$ and $0.1 y_{\max }, j=41$, and under an academic context of a fixed, non-overlapping dense multipath channel [19] that can have stronger MPCs arriving after the direct path. As shown, the results match well with each other which further signifies the importance of threshold setting according to the desired $P_{F A}$ and $P_{M}$. Furthermore, a higher $\xi$ tends to decrease the $P_{F A}$ whilst increasing the $P_{M}$, and vice versa for a lower $\xi$, which is intuitively correct. However, due to the difficulty in obtaining the exact $a_{i^{\prime}}$ from the noise-corrupted observation, the evaluation of (28) in reality is still quite challenging, and for the simplest of all selection criteria one would have to resort to the setting provided by (25). Note that the noise variance that is required to determine the threshold in the above formulations can be estimated according to (15).

\section{Results}

We compare the performance of the RLS-WD with the suboptimal ML PDB estimators in [9], namely, single search (PDB-SS1), search and subtract (PDB-SS2), search subtract and readjust (PDB-SSR). In general, these estimators first compute the discrete match filter (MF) output between $\mathbf{r}$ and a sampled pulse template; thereafter, the selection of the maximum MF peaks and, depending on the algorithm, with or without iteratively computing the MF output after removing the peaks from $\mathbf{r}$. Performance is examined in 


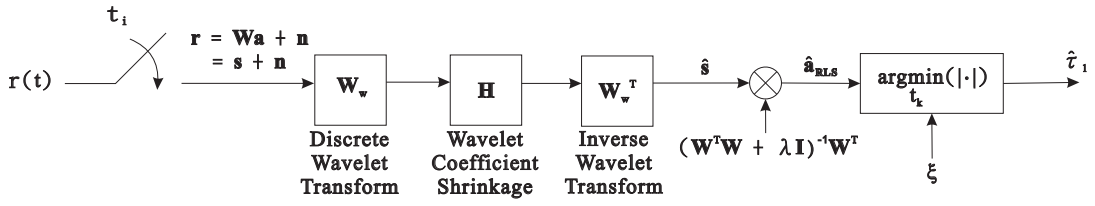

Figure 1: The RLS-WD ToA estimator.
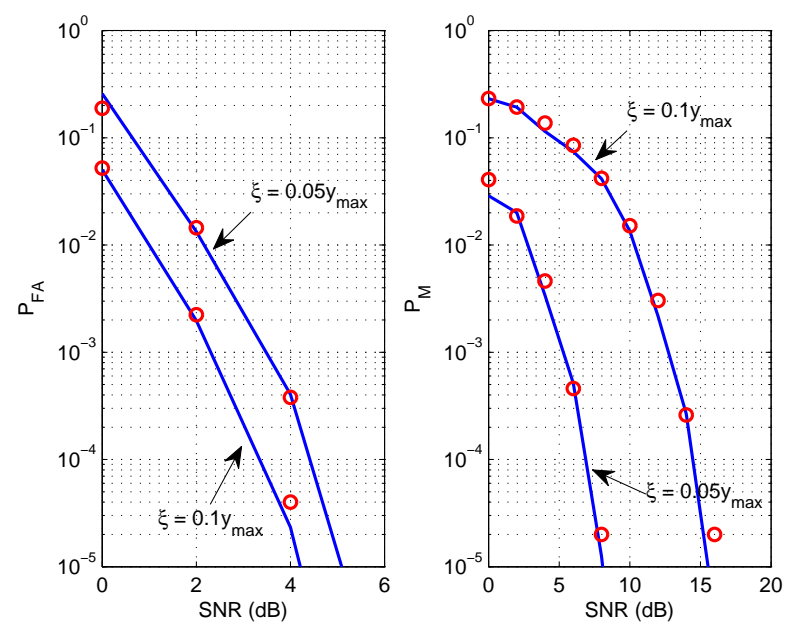

Figure 2: Comparison of theoretical and simulation results for both $P_{F A}$ (left) and $P_{M}$ (right) as a function of SNR under two fixed thresholds $\xi$ of $0.05 y_{\max }$ or $0.1 y_{\max }, j=$ 41 , and with a fixed, non-overlapping dense multipath channel that can have stronger MPCs arriving after the ToA. The lines and circles are theoretical and simulation results, respectively. 
three categories, i.e., via numerical simulation, with realistic indoor measurement data, and via a complexity analysis.

\subsection{Numerical Simulation}

We evaluate the performance by computer simulation in MATLAB ${ }^{\mathrm{TM}}$ with the Uvi_Wave software package [20] for Daubechies DWT. To accurately examine the performance under multipath, we use the CM3 channel model from IEEE 802.15.3a [19], which models a severe office NLOS environment. The received template $w(t)$ is assumed to be the typical Gaussian doublet with pulse parameter $\tau_{m}=0.6 \mathrm{~ns}$, which has a zero-to-zero pulse width of $2 \mathrm{~ns}$. The pulse is sampled at $T_{s}=0.1 \mathrm{~ns}$ with the observation interval $T=50 \mathrm{~ns}$, representing a medium distance ranging application. To study the performance of RLS-WD ToA estimator, we vary $\Delta$ as a multiple of $T_{s}$ when constructing $\mathbf{W}$. A thousand different channel realizations are simulated prior to the final performance evaluation. For the PDB algorithms, the number of peaks to detect $Z$ is set to 100 . Since the iterative PDB algorithms have no known optimal threshold selection criterion [9], we employ a fixed threshold when comparing the algorithms instead of adhering to the threshold selection criterion of Section 5 .

To determine the most suitable $F$ for WD, we plot the output SNR as $F$ varies for a fixed input SNR5 of $0 \mathrm{~dB}$ in Fig. 3(a). As shown, by applying WD to the received signal we can have close to $4 \mathrm{~dB}$ gain across all $F$. Since increasing $F$ has no effect on the output SNR, we may further reduce the denoising complexity by selecting the smallest $F$ before performance tapers off. For $F=8$, Fig. 3(b) illustrates the effectiveness of WD6 as the input SNR varies. We see that WD results in substantial gain at low SNR before diminishing return at high SNR. However, when considering long distance ranging, the performance at low SNR is often of great interest. For the ease of simulation, $\sigma^{2}$ in (13) is assumed to be perfectly known, hence the results shown act as a lower bound. However, in the next section we show that even when the noise variance is estimated with (15), the gain of denoising at low SNR is still substantial.

\footnotetext{
${ }^{5}$ The output and input $\mathrm{SNR}$ are defined as $\mathrm{SNR}_{\text {out }}=20 \log _{10}(\|\mathbf{s}\| /\|\hat{\mathbf{s}}-\mathbf{s}\|)$ and $\mathrm{SNR}_{\text {in }}=$ $20 \log _{10}(\|\mathbf{s}\| /\|\mathbf{n}\|)$, respectively, where $\|\cdot\|$ denotes the Frobenius matrix norm [12]. In all of the later simulations, we set the SNR according to this $\mathrm{SNR}_{\text {in }}$ definition.

${ }^{6}$ The effectiveness of WD can be measured from the gain in SNR, i.e., $\mathrm{SNR}_{\text {out }}-\mathrm{SNR}_{\text {in }}$.
} 


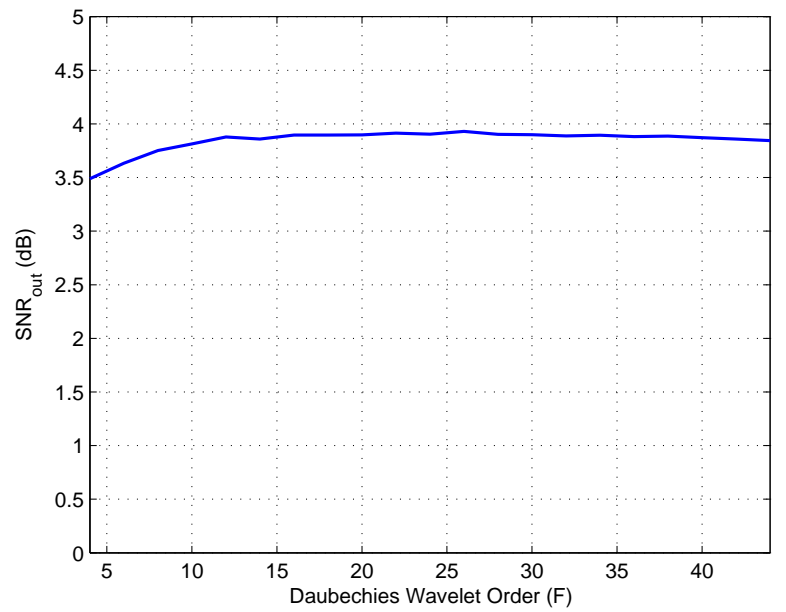

(a) SNR of denoised signal as $F$ varies for $0 \mathrm{~dB}$ input SNR.

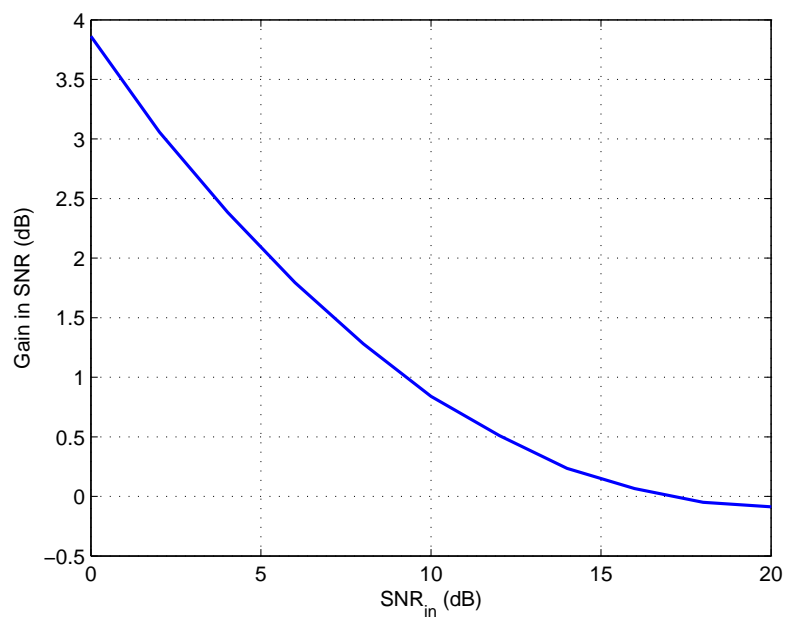

(b) Gain in SNR as a function of input SNR for $F=8$.

Figure 3: Denoising performance of Daubechies DWT for UWB-IR. 
Fig. 4 shows the behavior of root mean-squared error (RMSE) versus SNR when $\xi$ is fixed at $0.05 y_{\max }$, with the RLS-WD evaluated at $\lambda=2$ in (9), and $\Delta=2 T_{s}$ and $4 T_{s}$. Clearly, the RLS-WD algorithm outperforms others under all SNR. At low SNR, it performs better due to the input SNR enhancement from WD; whereas, at high SNR, the contribution from WD lessens. An interesting observation is the identical performance for $\Delta=$ $2 T_{s}$ and $4 T_{s}$, which depending on the system requirement we may choose either one without sacrificing the overall performance. We observe a similar improvement on the RMSE when $\xi$ is fixed at $0.1 y_{\max }$, as shown in Fig. 5 . Specifically, the RLS-WD outperforms PDB algorithms when SNR $<15 \mathrm{~dB}$. For $\mathrm{SNR} \geq 15 \mathrm{~dB}$, all algorithms exhibit an error floor, particularly a higher floor for RLS-WD than PDB-SSR, which is due to a combination of the threshold setting and the channel characteristic of stronger paths arriving after the first path arrival, thus increasing the error floor. Once again, we stress on the importance of performance gain at low SNR that is more crucial in long distance ranging application.

Apart from the RMSE behavior, we are also interested in the energy capture, as in [9]. For that, Fig. 6 illustrates the mean energy capture as a function of SNR for all algorithms, and the RLS-WD is computed with $\lambda=0.1$. The energy capture is computed between the received signal and its estimate. From Fig. 6 we note that the RLS-WD loses energy initially due to denoising, but it quickly recovers at high SNR when the received signal is less noisy. Also, a spacing of $2 T_{s}$ on $\Delta$ captures significantly more energy than for $\Delta=4 T_{s}$ since $K$ decreases as we increase $\Delta$ in the signal model. Note that if all algorithms undergo denoising before ToA estimation, the energy capture of RLS-WD would still outperform its counterparts.

Without the threshold selection criterion as described in Section 5, the choice of a fixed $\xi$ can have a pronounced effect on the estimator performance. For that, we illustrate the effect of a varying $\eta$ with a fixed $y_{\max }$ has on the RMSE in Fig. 7 at $\Delta=2 T_{s}$ for $\mathrm{SNR}=\{0,4,8,12,16,20\} \mathrm{dB}$. Generally, a small $\eta$ does not produce the best result, especially in the low SNR environment where noise can often be mistakenly identified as the direct path. However, at high SNR, a low $\eta$ often produces the best result since the noise is either small or has been mostly removed by WD.

\subsection{Propagation Measurements}

We evaluate the four algorithms under the realistic application of indoor

ranging with the UWB measurements from [4]. A short description of the 


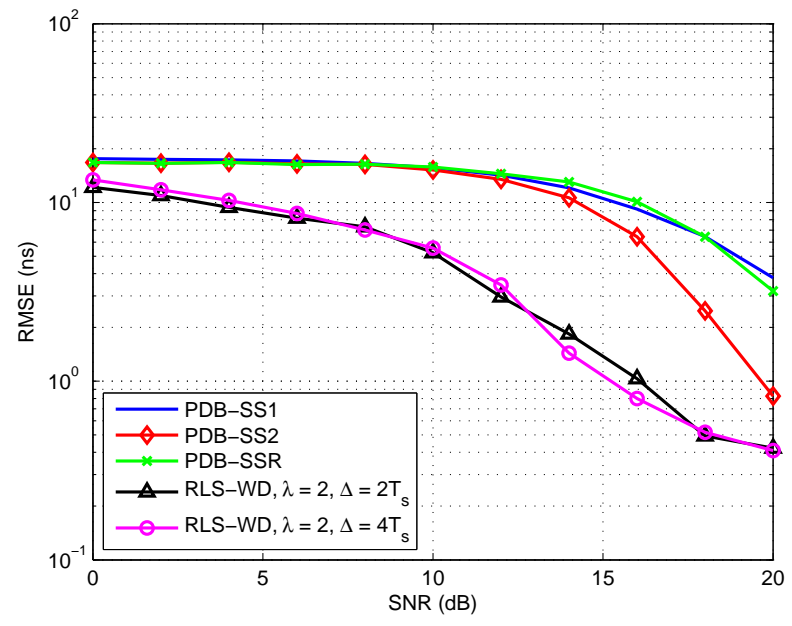

Figure 4: RMSE of ToA estimation as SNR varies for different algorithms with a fixed $\xi=0.05 y_{\max }$.

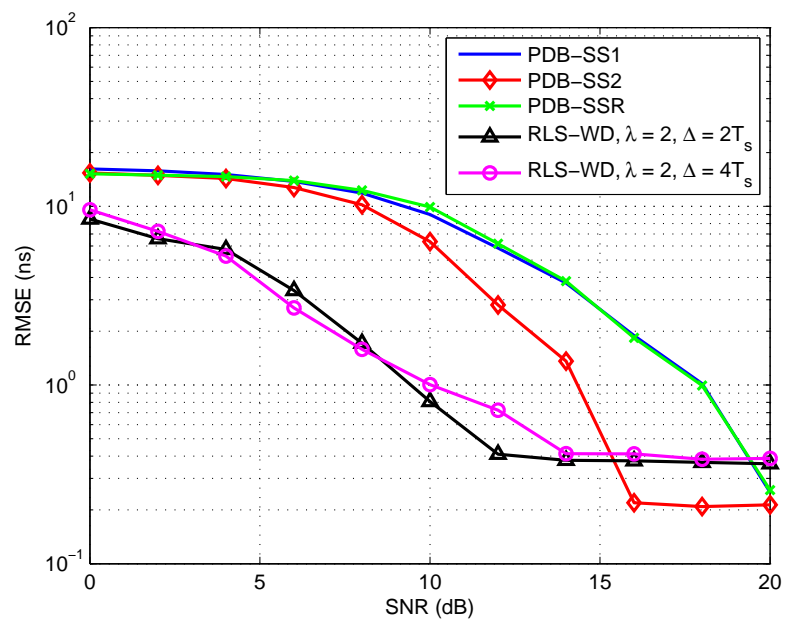

Figure 5: RMSE of ToA estimation as a function of SNR for different algorithms with a fixed $\xi=0.1 y_{\max }$. 


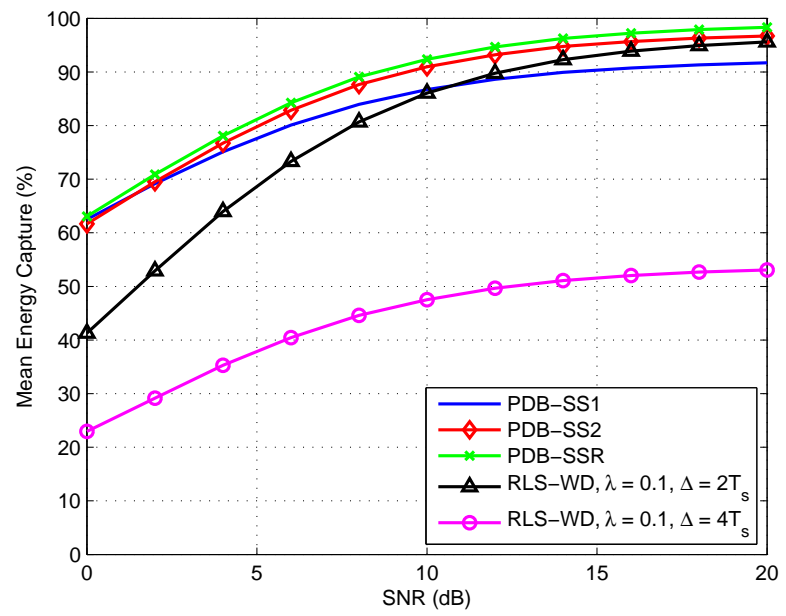

Figure 6: Mean energy capture as SNR varies for different algorithms. The energy capture is computed between the received signal and its estimate.

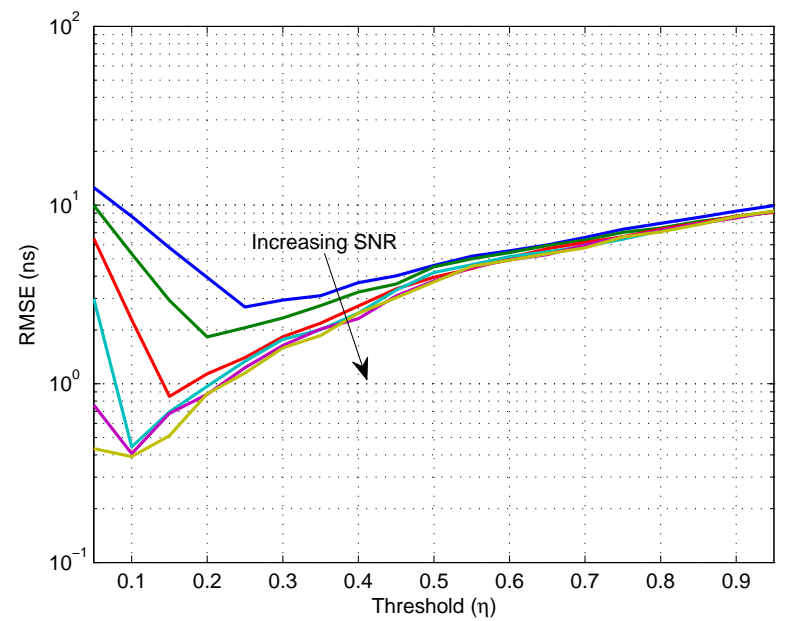

Figure 7: RMSE versus $\eta$ for the RLS-WD ToA estimator at $\Delta=2 T_{s}$ for SNR $=$ $\{0,4,8,12,16,20\} \mathrm{dB}$, top to bottom. 
experiment is in order. The measurements were taken in a laboratory environment where the interior walls are made of metal stud and dry wall with either cinder blocks or wooden constructions. Fig. 8 shows the building floor plan where measurements of up to 93 feet were taken. Except the clear LOS reference at location 1 , the remaining signals were recorded with a multitude of obstructions in between the transmitter (TX) and the receiver (RX), resembling NLOS operation. The setup consisted of a pulser that periodically transmits a sub-nanosecond pulse to the RX side, which then amplifies the received signal before being sampled by a digital sampling oscilloscope for post-processing offline. A pair of vertically polarized diamond dipole antennas were used to radiate the signal. We apply WD in Fig. 9 to the measured signal at location 13. Clearly, even with $\hat{\sigma}^{2}$ computed 7 according to (15), the noise can be mostly removed from the contaminated signal without much signal distortion.

Fig. 10 compares the range estimation errors at each location of Fig. 8 for a fixed $\xi=0.1 y_{\max }$, between (a) the different PDB algorithms with an optimized $Z$, and (b) the RLS-WD with different $\Delta$. The error is computed as the difference between the estimated and measured ToA, assuming there exists a clear LOS path between the TX and RX for the direct-path signal [4]. As shown, optimizing $Z$ for the PDB algorithms significantly reduces the range estimation error at short distances. Despite the optimization, PDBSS1 always produces the worst performance at long distances followed by the PDB-SS2 and the PDB-SSR, as in agreement with the simulation results of Section 6.2, which is due to the inaccuracy in delay estimation with the picking of $Z$ largest peaks after only one MF correlation; whereas, both PDBSS2 and PDB-SSR readjust their delays at each iteration for an improvement in accuracy. Altogether, we see that the RLS-WD performs better for most cases at long distances, and can perform better or equal to that of the PDB algorithms at short distances. We would like to stress that whereas one may need to switch between different PDB algorithms for the best performance under all circumstances, we would only need to store $\mathbf{W}$ with different $\Delta$ in a memory bank for rapid signal processing. As noted in [4], the large errors at long distances are mostly caused by a combination of the complex LOS

\footnotetext{
${ }^{7}$ In (15), the $N$ samples of $M$ are obtained from the first few nanoseconds of the measurements until the direct-path ToA, which is calculated relative to the true measured range assuming a clear LOS path between TX and RX. In reality, $\sigma^{2}$ can be estimated from the samples when there is no communication between the nodes.
} 


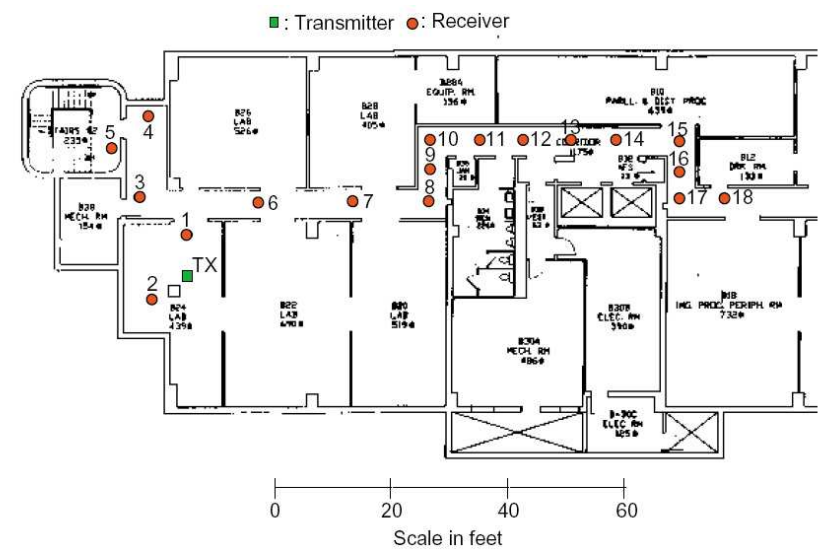

Figure 8: The building floor plan where the range measurements were taken by Lee in [4]. The circular and square marks indicate the location of the RX antenna and that of the TX antenna, respectively.
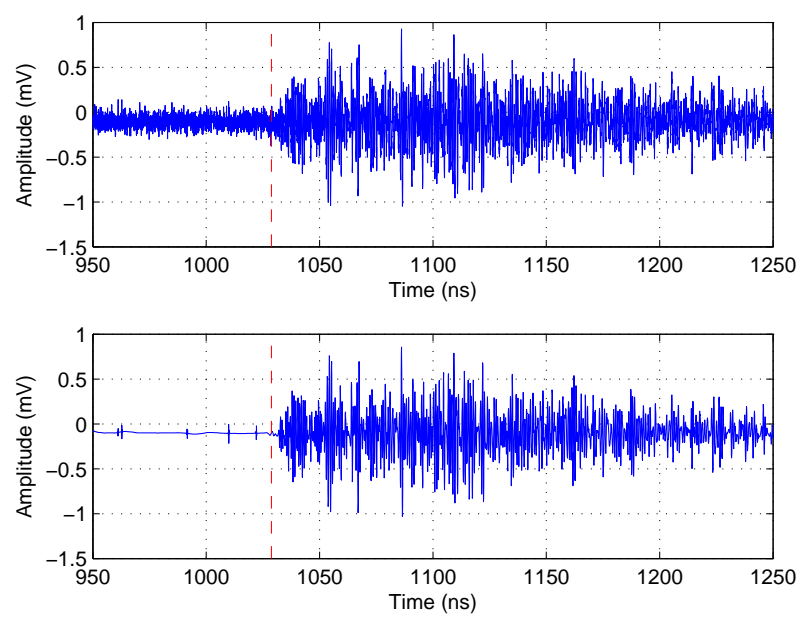

Figure 9: The measured signal taken at location 13 (top) and its denoised version (bottom). The vertical line of each plot denotes the direct-path ToA, assuming a clear LOS path between TX and RX in the true measured range. 
blockage and a systematic error in distance measurement, the investigation of which is beyond the scope of this paper.

Last but not least, we illustrate the effect of a varying $T$ has on the range estimation error in Fig. 11 for a fixed $\xi=0.1 y_{\max }$ and measurement at location 15, depicting a severe NLOS environment. As we shall see in the next section, the observation window $T$ plays a crucial role in determining the overall system complexity since it governs the number of samples to process in both the MF calculation and WD. With $Z=100$, the PDB algorithms tend to produce significant errors when $T$ is small such that the estimated channel taps fall into the noise region. In contrast, due to the linear system model assumption, the RLS-WD with a LS solution does not have this problem and can better estimate the direct-path arrival even with a small $T$, e.g., $T<150$ ns. This characteristic is similarly observed at all other locations. Therefore, comparing to its counterparts, the processing time of the RLS-WD can be dramatically reduced since we would only need to include the samples until the direct-path arrival, but nothing after.

\subsection{Complexity Analysis}

The computational load of the RLS-WD is mostly constant since most of the matrix operations in (5), (17), and (9) can all be done beforehand. Specifically, the RLS is equivalent to LS over a sphere and its complexity is about $4 M^{2} K+22 K^{3}$ flops [21] with the remaining load in DWT, which is of $\mathcal{O}(M)$ per WD process [22]. However, when recognizing the WD as a series of matrix multiplications in (14) with pre-computed matrices further indicates a constant processing time for our approach. In contrast, the complexity of the PDB estimators depends entirely on the channel condition and the number of iterations $Z$. Table 1 compares the flop count amongst the algorithms. For the $\mathrm{PDB}$ algorithms, ignoring the peak finding operations after the MF output, they require $4 z M^{2}$ flops per MF computation, $5 z M$ and $2 z^{2} M+$ $2 z^{3} / 3+2 z M$ flops to compute the channel gain per iteration for PDB-SS2 and PDB-SSR, respectively, where $z$ denotes the iteration index. Hence, in a dense multipath channel, the RLS-WD would require less processing time than its counterparts.

\section{Conclusion}

In this correspondence, we have proposed the RLS-WD ToA estimator, which estimates the ToA as a by-product of the RLS channel estimator based 


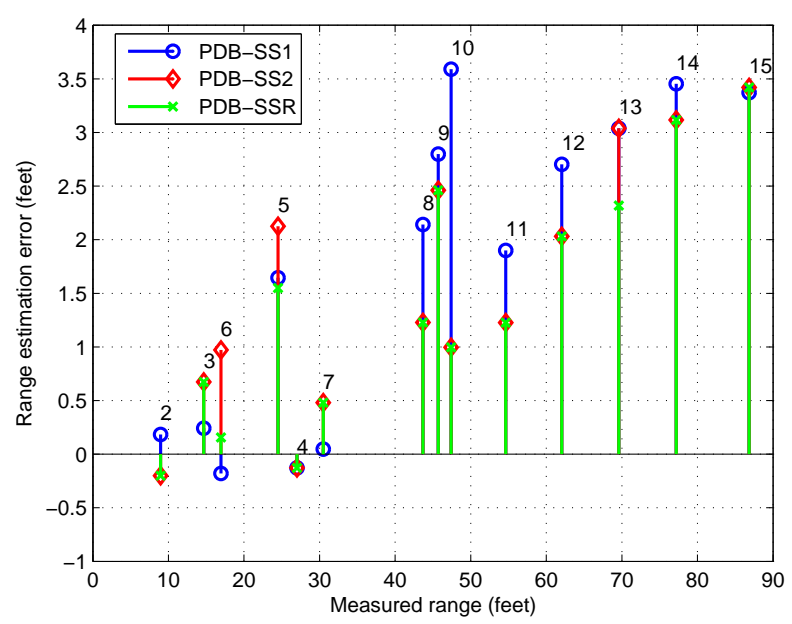

(a) PDB algorithms

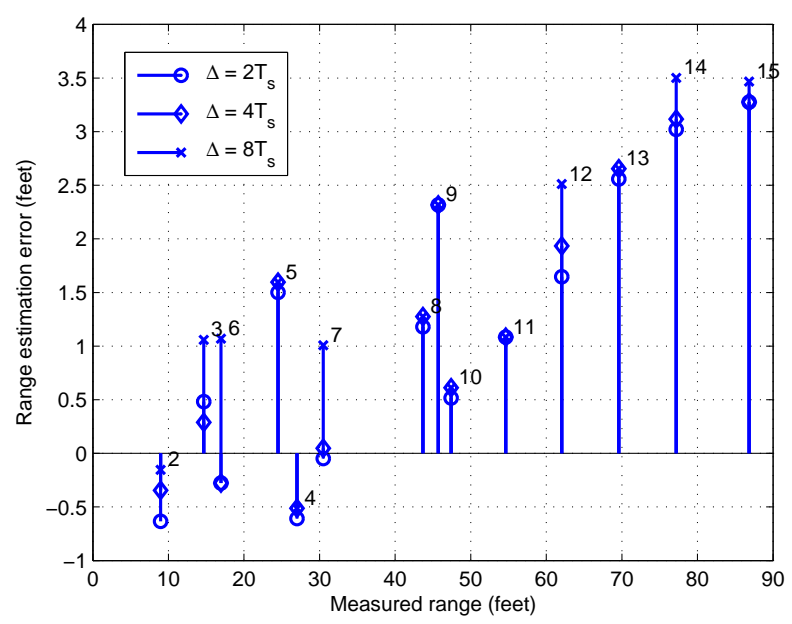

(b) RLS-WD algorithm with different $\Delta$

Figure 10: Comparison of the range estimation errors between the different algorithms with a fixed $\xi=0.1 y_{\max }$ at the locations in Fig. 8. For the PDB algorithms, the minimum error has been optimized according to $Z$ and the RLS-WD is evaluated with different $\Delta$ and $\lambda=2$. The error is calculated as the difference between the estimated and measured ranges, assuming the direct-path signal arrives via a clear LOS path between the TX and RX. 


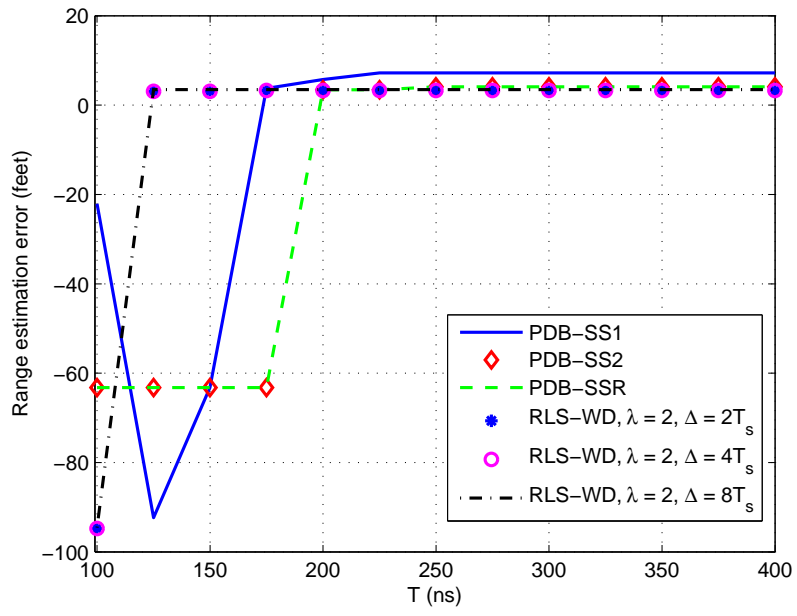

Figure 11: Comparison of range estimation error as observation window $T$ varies in step of $25 \mathrm{~ns}$ from $100 \mathrm{~ns}$ to $400 \mathrm{~ns}$ between the algorithms for the measurement taken at location 15. The parameters are $\xi=0.1 y_{\max }, \lambda=2, F=8$ and $Z=100$.

Table 1: Comparison of computational complexity between different ToA algorithms

\begin{tabular}{||c|c||}
\hline & Flop Count \\
\hline PDB-SS1 & $4 M^{2}$ \\
\hline PDB-SS2 & $4 Z M^{2}+5 Z M$ \\
\hline PDB-SSR & $4 Z M^{2}+4 Z M+\mathcal{O}\left(Z^{4}\right)$ \\
\hline RLS-WD & $\left(4 M^{2} K+22 K^{3}\right)+4 F J M$ \\
\hline
\end{tabular}


on a thresholding technique. Our approach is simple and can provide fast, on-the-fly, accurate ToA estimation applicable to real-time ranging system. Unlike the majority of the previous works, we have illustrated the robustness of our algorithm first by computer simulation and then reaffirmed with the processing of realistic indoor UWB ranging data. In both cases when sampling rate is available, our approach is shown to outperform other highresolution algorithms in ToA estimation, energy capture and computational complexity. The low-complexity and high accuracy nature of the RLS-WD ToA estimator will be an indispensable part of a real-time system when the instantaneous position estimate of a TN in a WSN is in high demand.

\section{Acknowledgment}

The authors would like to thank the UltRa Lab at the University of Southern California for providing the range measurements herein.

\section{References}

[1] S. Gezici, Z. Tian, G. Giannakis, H. Kobayashi, A. Molisch, H. Poor, Z. Sahinoglu, Localization via ultra-wideband radios: a look at positioning aspects for future sensor networks, IEEE Signal Processing Mag. 22 (4) (2005) 70-84. 2

[2] K. Pahlavan, X. Li, J. Makela, Indoor geolocation science and technology, IEEE Commun. Mag. 40 (2) (2002) 112-118. 2

[3] M. Win, R. Scholtz, Characterization of ultra-wide bandwidth wireless indoor channels: a communication-theoretic view, IEEE J. Select. Areas Commun. 20 (9) (2002) 1613-1627. 2, 3]

[4] J. Lee, R. Scholtz, Ranging in a dense multipath environment using an UWB radio link, IEEE J. Select. Areas Commun. 20 (9) (2002) 16771683. 2, 3, 9, 12, 17, 20, 21,

[5] I. Guvenc, Z. Sahinoglu, Threshold-based TOA estimation for impulse radio UWB systems, in: Proc. of IEEE ICUWB, 2005, pp. 420-425. 3

[6] S. Gezici, Z. Sahinoglu, A. Molisch, H. Kobayashi, H. Poor, A TwoStep Time of Arrival Estimation Algorithm for Impulse Radio Ultra Wideband Systems, in: Proc. of European Signal Processing Conference (EUSIPCO), 2005. 3 
[7] R. Balakrishnan, T. Gyawali, H. Kwon, An inverse problem based approach for channel parameters estimation in UWB systems, in: Proc. of IEEE Vehicular Technology Conference (VTC), Vol. 2, 2004, pp. 10731077. 3, 5

[8] Z. Low, J. Cheong, C. Law, W. Ng, Y. Lee, Pulse detection algorithm for line-of-sight (LOS) UWB ranging applications, IEEE Antennas Wireless Propagat. Lett. 4 (2005) 63-67. 3

[9] C. Falsi, D. Dardari, L. Mucchi, M. Win, Time of Arrival Estimation for UWB Localizers in Realistic Environments, EURASIP J. Appl. Signal Processing (Special Issue on Wireless Location Technologies and Applications) 2006, article ID 32082, 13 pages. 3, 4, 5, 6, 13, 15, 17

[10] D. Donoho, J. Johnstone, Ideal spatial adaptation by wavelet shrinkage, Biometrika 81 (3) (1994) 425-455. 3, 6, 7]

[11] P. Ching, H. So, S. Wu, On wavelet denoising and its applications to time delay estimation, IEEE Trans. Signal Processing 47 (10) (1999) 2879-2882. 3

[12] R. Sathish, G. Anand, Wavelet Denoising for Plane Wave DOA Estimation By MUSIC, in: Proc. of IEEE TENCON '03, Vol. 1, 2003, pp. 104-108. 3, 15

[13] Y. Xue, J. Wang, Z. Liu, Application of Wavelet Array Denoising to ESPRIT Algorithm, in: Proc. of IEEE TENCON '05, 2005, pp. 1-4. 3

[14] B. Vidakovic, Wavelets and Bayesian statistics, Interface '94 (1994) 1518. 3, 7

[15] D. Donoho, Denoising by soft-thresholding, IEEE Trans. Inform. Theory 41 (3) (1995) 613-627. 3, 7, 8

[16] A. Tikhonov, V. Arsenin, Solutions of ill-posed problems, John Wiley \& Sons, New York, 1977. 6

[17] S. Kay, Fundamentals of Statistical Signal Processing: Detection Theory, Prentice-Hall, Upper Saddle River, NJ, 1998. 9

[18] A. Papoulis, Probability, random variables, and stochastic processes, McGraw-Hill, New York, 1991. 9, 10, 
[19] A. Molisch, J. Foerster, M. Pendergrass, Channel models for ultrawideband personal area networks, IEEE Wireless Commun. Mag. 10 (6) (2003) 14-21. 13, 15,

[20] S. Sanchez, N. Prelcic, S. Galan, Uvi_Wave, Grupo de Teorıa de la Senal, Universidad de Vigo. 15

[21] G. Golub, C. Van Loan, Matrix computations, 3rd Edition, The Johns Hopkins University Press, 1996. 22

[22] M. Vetterli, J. Kovačević, Wavelets and subband coding, Prentice-Hall, Englewood Cliffs, NJ, 1995. 22 